\title{
A Generic Information Service Framework for Physically Challenged People in Wireless Networks
}

\author{
Kiran Kumari Patil, Member, IACSIT, Pramod, Shrihari, Vijay Kumar B. P, Member, IACSIT, \\ and T. N. Nagabhushan
}

\begin{abstract}
Design of ubiquitous technology for providing an unobtrusive environment for day to day routine activities for physically challenged people is a challenging issue. More reliable system with accurate information dissemination depending on the context location is important in the design of such systems. In this work we have proposed a model using mobile location based information dissemination for physically challenged persons (hearing/visually impaired persons). An audio/video assistance application is developed, that provides the directional information vector at any instant on the handheld device. The contextual information is obtained from the connectivity, orientation of device, person's impairment, time, location, etc. The prototype implementations are carried out at our research lab, using recently released Internet mobile phone/tablet (N810, N900), and using open source Maemo platform. Suitable GUI is developed for voice / video information dissemination. Applications are developed by mapping our institution layout to access point position and orientation of the mobile device.
\end{abstract}

Index Terms-Physically Challenged, Location, Mobile Device, Meamo, WiFi, Internet, GPS.

\section{INTRODUCTION}

Physically challenged people's major setback is that they have to depend on the others, for their day to day routine works. The proposed mobile based computing model helps to overcome their problems by providing a reliable and real time location and context based information using hand held mobile devices. The novelty in the proposed model involves in developing a computing algorithm for finding wireless connectivity, location of mobile device, orientation, mapping database to the respective queries by the users. Wifi routers are used to display at proper position in a given area to have a proper networking infrastructure depending on organizational and its layout. Using signal power measurement and techniques mobile locations are identified to provide the directional information to user along with other contextual parameters. In our model we considered time, orientation, personal impairment as contextual parameters to generate directional information to the user. Prototype implementation and testing is done. Open source platform like Maemo, Qt are used in the portable device for development of application called PCPA (Physically Challenged Personal Assistance).

Rest of the paper is arranged accordingly, in section 2 discussions over related work is done. Hardware and

Manuscript received June 22, 2010; revised July 6, 2011.This work was supported by NOKIA - VTU Collaborative Research, Reva ITM, Bangalore, India.

Kiran Kumari Patil is with REVA ITM, Bangalore, India software requirements are discussed in section 3. The existing system details are highlighted in section 4 . Section 5 is concerned with the proposed model. The prototype implementation of the proposed model is explained in section 6. Concluding high lights are given in section 7.

\section{RELATED WORK}

A number of approaches have been explored to help blind travelers with orientation, navigation and wayfinding, most using modalities other than computer vision. Among the most promising include infrared sign age that broad-casts information received by a hand-held receiver [3], GPS-based localization(e.g.http://www.senderogroup.com), RFID labeling, and indoor Wi-Fi based localization (based on signal strength) and database access [2]. However, each of these approaches has significant limitations that limit their attractiveness as stand-alone solutions. Infrared signs require costly installation and maintenance; GPS has poor resolution in urban settings and is unavailable indoors; RFIDs can only be read at close range and would therefore be difficult to locate by blind travelers; and Wi-Fi localization requires extensive deployment to ensure complete coverage, as well as a time-consuming calibration process. Research has been undertaken on computer vision algorithms to aid in wending for such applications as navigation in traffic intersections [5] and sign reading [4]. The obvious advantage of computer vision is that it is designed to work with little or no infrastructure or modification to the environment. However, none of it is yet practical for commercial use because of issues such as insufficient reliability and prohibitive computational complexity (which is especially problematic when using the kind of portable hardware that these applications require). Our approach, Location-based service, is more user requirement specific and context based approach makes PCPA application to solve the major challenge of physically impaired person.

\section{HARWARE AND SOFTWARE}

The application is being developed for Nokia N810/N900. It is a mobile Internet device and Smart phone from Nokia that supersedes the N810. Based on the Maemo platform, it runs Maemo 5 Linux as its default operating system and is the first Nokia device based upon the TI OMAP3 microprocessor with the ARM Cortex-A8 core. Unlike the Internet Tablets preceding it, the Nokia N900 is the first Maemo device to include phone functionality (quad-band GSM and 3 G UMTS). It functions as a 5 mega pixel camera, 
a portable media player, and a mobile Internet device with email and full web browsing.

Maemo is a software platform developed by the Maemo community for smart phones and Internet tablets. It is based on the Debian Linux distribution. The platform includes the Maemo operating system and the Maemo SDK. Maemo is mostly based on open source code, and has been developed by Maemo Devices within Nokia in collaboration with many open source projects such as the Linux kernel, Debian, and GNOME. Maemo is based on Debian GNU/Linux and draws much of its GUI, frameworks, and libraries from the GNOME project. It uses the Matchbox window manager, and the GTK-based Hildon as its GUI and application framework.

The application is developed using Qt. A cross-platform application and UI framework. It includes a cross-platform class library, integrated development tools and a cross-platform IDE. Using Qt, we can write web-enabled applications once and deploy them across many desktop and embedded operating systems without rewriting the source code. SQLite is the database system used. SQLite is an ACID-compliant embedded relational database management system contained in a relatively small $\mathrm{C}$ programming library. The source code for SQLite is in the public dominant implements most of the SQL standard. In contrast to other databases, SQLite is not a separate process that is accessed from the client application, but an integral part of it.

\section{A. . Existing System}

The Global Positioning System (GPS) is a space-based global navigation satellite system that provides reliable location and time information in all weather and at all times and anywhere on or near the Earth where there is an unobstructed line of sight to four or more GPS satellites. It is maintained by the United States government and is freely accessible by anyone with a GPS receiver.

The GPS System was created and realized by the U.S. Department of Defense (DOD) and was originally based on and run with 24 satellites. It was established in 1973 to reduce the large number of navigation aids and to overcome the limitations of previous navigation systems.

A GPS receiver calculates its position by precisely timing the signals sent by the GPS satellites high above the Earth. Each satellite continually transmits messages which include

1) The time the message was transmitted

2) precise orbital information (the ephemeris)

3) The general system health and rough orbits of all GPS satellites (the almanac).

The receiver utilizes the messages it receives to determine the transit time of each message and computes the distances to each satellite. These distances along with the satellites' locations are used with the possible aid of trilateration, depending on which algorithm is used, to compute the position of the receiver. This position is then displayed, perhaps with a moving map display or latitude and longitude; elevation information may be included. Many GPS units show derived information such as direction and speed, calculated from position changes. Three satellites might seem enough to solve for position, since space has three dimensions and a position near the Earth's surface can be assumed. However, even a very small clock error multiplied by the very large speed of light - the speed at which satellite signals propagates results in a large positional error. Therefore receivers use four or more satellites to solve for the receiver's location and time. The very accurately computed time is effectively hidden by most GPS applications, which use only the location. A few specialized GPS applications do however use the time; these include time transfer, traffic signal timing, and synchronization of cell phone base stations. Although four satellites are required for normal operation, fewer apply in special cases. If one variable is already known, a receiver can determine its position using only three satellites. (For example, a ship or plane may have known elevation.) Some GPS receivers may use additional clues or assumptions (such as reusing the last known altitude, dead reckoning, inertial navigation, or including information from the vehicle computer) to give a less accurate (degraded) position when fewer than four satellites are visible.

\section{B. Limitations of GPS}

The major limitation of the existing GPS based navigation technology looked into, in our project is that the GPS technology remains ineffective in a closed environment such as a commercial complex or a college building. Extending on the limitation stated above the following system is proposed.

\section{The Proposed MOdel}

PCPA application provides the context based directional information on the mobile devices for disabled persons. A high level architecture of a PCPA application is as shown in figure 1. Access points will be deployed in the entire building, when a person enters the premises, gets connected to the buildings Wifi network. Based on the contextual information (such as location, orientation, persons kind of impairment, time etc), directional information will be provided.

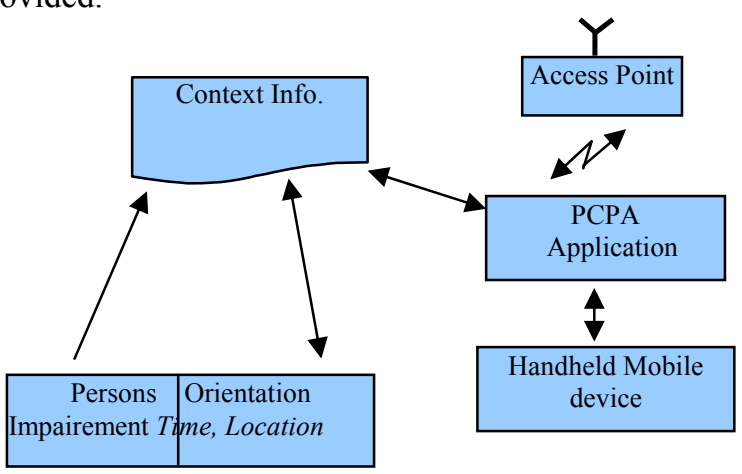

Fig. 1. Architecture of PCPA Model.

\section{A. Features of the Proposed System are}

1) Providing the location information on the mobile devices designed for disabled people with a touch screen, voice processor and other contextual information can be disseminated with proper aggregation.

2) Development of support for decision making.

3) Implementing it on a portable device the system deals with dynamic updating of information and provided to 
the users.

4) Information provided is not general information it provides information based on the nature of the land so location based specific information is provided.

5) Maintenance is easier there is no requirement of a single individual to work on the maintenance of the system.

\section{Prototype Implementation Of The Proposed Model}

The major focus of our project is indoor navigation. The most common and widely available form of signal available inside a building is Wi-Fi signal. Our project works on this wide availability of Wi-Fi signal. The concept of GPS navigation is borrowed here but instead of GPS signal Wi-Fi signals are used. Usage of Wi-Fi signal makes the framework developed more portable. This concept of using Wi-Fi signals for indoor navigation is further personalized to the specific needs of a visually challenged person. This can be achieved by integrating audio based user interface to the developed framework.

This project framework helps such people to overcome their problems with the help of an Internet Tablet (or Wi-Fi enabled device). Internet Tablets fall in the range between PDAs and focus on internet and media features.

The internet tablet makes use of the internet packets sent from the Wi-Fi routers installed in the building. It gets the co-ordinates of the location using the GPS in the device and puts that on the virtual map of the building giving the precise location of the person holding the device. It can also be displayed in various languages. This location is then converted to speech for the purpose of ease.

Maemo 5, SDK platform is used for the development of PCPA application. The network connectivity with in the laboratory is established by using Wifi access points, with servers having all the mapping information and database related to geographical location, building infrastructure, and orientation of the mobile with respect to the user handling the mobile device. Implementations can be carried out in two ways. One is, Client server model, with client process running on mobile device and server process on a fixed computing device connected to the LAN along with Wifi access points.

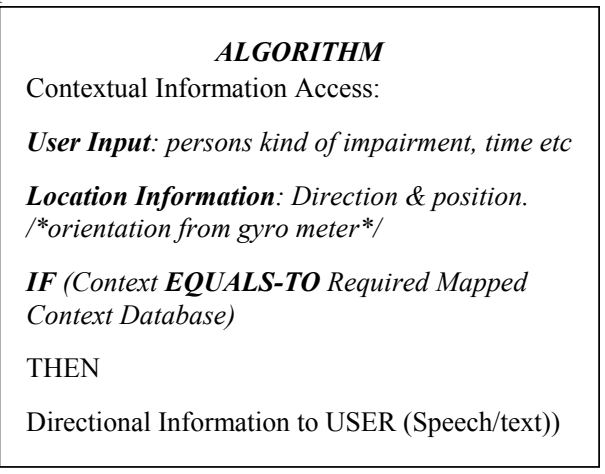

Fig. 2. An Algorithm for Accessing required Data.

The client server computing will generate proper information based on the clients request, its position and orientation. The second method is a standalone computing in handheld device having the entire database and mapping information. Here the information dissemination happens based on the location of the handheld device identified through access point-ID and signal power orientation information, based on the algorithm, explained in figure 2. Here the information's are obtained through gyro meter parameters along with time, person's impairment and physical structure of the organization. The contextual information includes the environmental details like location identity, access methodology. User inputs are taken explicitly from the user with proper accessing Techniques Location Information is used to show the device location, identified by access point connectivity with handheld device and corresponding mapping. Context database includes the mapping table containing the information above the position of access point, layout planning of the organization and the current position of handheld device. As soon as the current position of the handheld is recorded as context information, the server will provide $\&$ send the computed directional information from the mapping table as shown below in figure 3 in terms of database model.

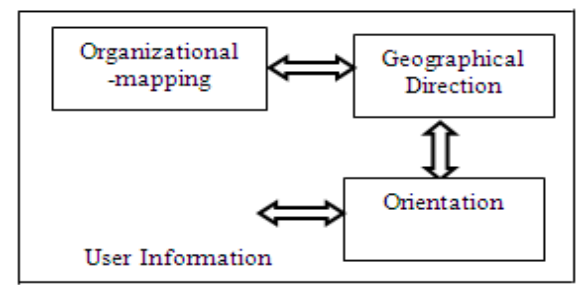

Fig. 3. Database Model for PCPA application.

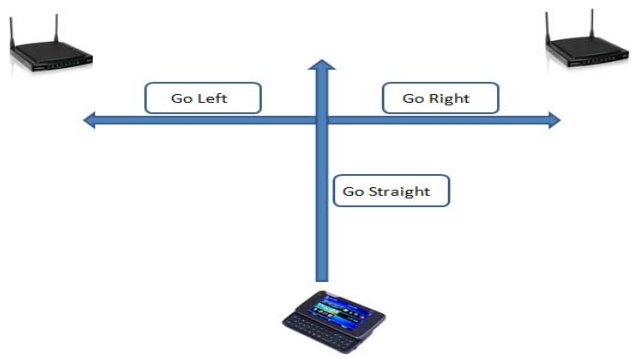

Fig. 4. Experimental setup

\section{A. Activities Involved in the proposed system}

1) Scanning the Wi-Fi signals, comparing them along with the distance of the user from the router and getting the location based on this data.

2) Pointing out the location on the virtual map generated on the device for an added functionality.

3) Converting the text to speech for an added functionality.

4) Aggregation is a method where, the information is put together from various sources and it is carried forward for dissemination.

5) Dissemination is a method where, the information from Aggregation is collected and put forward to be received by the client mobile devices.

The required components and experimental setup for PCPA is as shown in the above figure 4. Following are the components used for the development of this framework:

1) Wi-Fi routers ( two or more )

2) Wi-Fi enabled devices (n900/ N810 internet tablets)

3) Information Server (optional) 


\section{B. Wi-Fi Routers}

These are the devises that are used to enable wireless internet access. The technology used is IEEE 802.11 and the variants are $\mathrm{a} / \mathrm{b} / \mathrm{g}$. The data rate depends on the variant of IEEE 802.11 technology used. The major focus however is not on the data rate but the signal strength emitted by these routers. The strength of the signal is looked into and the entire navigation is based on the signal and position of these routers. For effective operation of the developed framework, a minimum of 3 routers are required. Its operational effectiveness in terms of distance, direction, and accuracy increases as the number of routers increases.

\section{Wi-Fi enabled devices}

These are handheld devices, like in our development we have used N810 / N900 that are Wi-Fi enabled. The signals emitted by the routers that are Wi-Fi enabled are read by these mobile devises. The applications are developed on these devises, to read the signal strength and identify the probable distance and location of the device, and hence the position of the user holding the device.

\section{Navigation through $\mathrm{Wi}-\mathrm{Fi}$}

The limitation mentioned above about the GPS based navigation technology can be strengthened by using Wi-Fi. The signals emitted by the $\mathrm{Wi}-\mathrm{Fi}$ access points is used to track the position of the mobile devices carried by the person and there by the person himself. The detailed implementation procedure is mentioned in the implementation section.

\section{E. Navigation assistance for physically challenged}

A physically challenged person's major setback is that he has to depend on the others. The dependency is so critical that they have to depend on others for every single work they do, for instance, to take a walk and other chores depending on their disabilities. This makes them mentally weak, makes them feel dependent and makes them un-confident.

The idea of using the Wi-Fi signals to track the person holding the Wi-Fi enabled devices can be further extended to help the disabled person to navigate through a closed environment where the GPS device cannot be used.

\section{F. Advantages of Proposed System}

1) Providing the location information on the mobile devices designed for disabled people with a touch screen, voice processor and other contextual information can be disseminated with proper aggregation

2) Development of support for decision making

3) Implementing it on a portable device the system deals with dynamic updating of information and provided to the users.

4) Information provided is not general information it provides information based on the nature of the land so location based specific information is provided.

5) Maintenance is easier there is no requirement of a single individual to work on the maintenance of the system.

\section{CONCLUSION}

PCPA is basic personal tool for physically challenged people. The proposed model is designed and developed on Qt - Maemo platform and deployed on the SDK for emulation and later tested on the hand held devices and Internet Tablets (i.e., N810, Nokia N900). The output of the prototype applications works favorably under ideal test conditions. The system can be used in any organizational infrastructure with proper signal conditions and Wi-Fi deployment planning for better resolutions to have directional vector information for the user and later helps a person to navigate within the organization. It assist a person to reach to specific location, within a building. Further work can be extended to voice based service helps a blind person in particular to find the location of his interest in the building.

\section{A. Future Enhancement}

Further enhancement of the application can be done by the inclusion of more number of routers for location and precise calculation of navigational information. Also providing a facility for loading the organizational maps dynamically, improves scalability. Several bugs that appear during non ideal condition are to be looked into before real time deployment of the application.

\section{ACKNOWLEDGMENT}

We would like to thank Mr. Suresh Chande, VTU-Nokia Relationship Manager from Finland for his technical support and guidance.

\section{REFERENCES}

[1] S. Chen, B. Mulgrew, and P. M. Grant, "A clustering technique for digital communications channel equalization using radial basis function networks," IEEE Trans. on Neural Networks, vol. 4, pp. 570-578, July 1993.

[2] Andrew S. Tanenbaum, Computer Networks, Prentice Hall Professional, 2003, ch 2, pp 100-117.

[3] Frank Ohrtman, konard roeder, WiFi Handbook: Building $802.11 \mathrm{~b}$ Wireles, McGraw-Hill Networking Professional, 2003, ch. 1, pp. 1-12, ch. 2, pp. 13-37.

[4] A.M. Ladd, K.E. Bekris, A.P. Rudys, D.S. Wallach and L.E. Kavraki. "On the feasibility of using wireless Ethernet for indoor localization”, IEEE Trans. On Robotics and Automation, June 2004, pp. 555-9.

[5] Jeff Duntemann, Jeff Duntemann's Drive - By Wi-Fi Guide, paraglyph Press, 2000, ch.1. pp. 3-20, ch.4. pp. 65-75.

[6] Bhawna Misra, Hand Book of Teaching for Physically Disabled, New Delhi, Mohit Publications, 2002, ch.1, pp 1-20.

[7] P. Silapachote, J. Weinman, A. Hanson, R. Weiss and M. A. Mattar. "Automatic Sign Detection and Recognition in Natural Scenes. ” IEEE Workshop on Computer Vision Applications for the Visually Impaired (CVAVI '05), in conjunction with CVPR '05. San Diego, CA. June 20, 2005

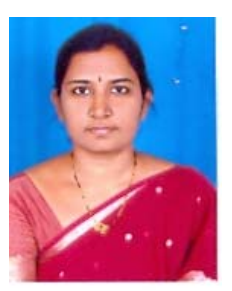

data retrieval.
Kiran Kumari Patil, is working as Assistant Professor at REVA ITM, Bangalore, India In the Department of Computer Science and Engineering.. She has received B.Tech, in Computer Science and Engineering and Masters in System Design and Technology from University of Applied Sciences, Darmstadt, Germany, she is currently pursuing Ph.D at Dr. MGR University Chennai University. Her main research interest includes Content Based Multimedia 